\title{
Human Communication Based on Icons in Crisis Environments*
}

\author{
Siska Fitrianie, Dragos Datcu, and Leon J.M. Rothkrantz \\ Man-Machine Interaction Group, Delft University of Technology \\ Mekelweg 4, 2628CD Delft, the Netherlands \\ \{s.fitrianie, d.dactu, l.j.m.rothkrantz\}@ewi.tudelft.nl
}

\begin{abstract}
In recent years, we have developed an icon-based communication interface to represent concepts and ideas. Users can create messages to communicate with others using a spatial arrangement of visual symbols. We deployed our icon-based interface in a serious game environment of a disaster and rescue simulator, which is capable of simulating real disaster situations using information from human user observers' reports. To support faster interaction, we designed a highly adaptive interface for optimizing the next icon look up. Inspired by the Fitaly keyboard, the system rearranges the icons menu's layout dynamically to minimize the searching time. Users are able to find their desired icons fast since the next icon selection is most likely to be one that is (on or) around the center. Our user tests showed that the developed iconbased interface could serve as a communication mediator. The experimental results also indicated that the Fitaly-based interface allowed for much faster and easier icon finding than the hierarchical menu.
\end{abstract}

\section{Introduction}

Nowadays, icons are used in almost every GUI-based computer software. Direct manipulation on the icons on a GUI allows us to have a fast interaction. Research showed that direct manipulation with a pointer has better time performance than form filling with Soft Input Panels or handwriting recognition [20]. As pictorial signs, icons can be recognized quickly and committed to human's memory persistently [12]. Therefore, icons can evoke a readiness to respond and quick ensuing actions [23].

Icons can form a language, where each sentence is created by a spatial arrangement of icons [4]. Each icon is understood as a representation of a concept, i.e. an object, an action, or a relation [27]. By virtue of resemblance between a given icon and the object or the movement it stands for, an icon functions as a mean of communication. Therefore, icons offer a potential across language barriers and a direct method for conversion to other modalities. Since icons are representations of models or concepts, with which humans are actually interacting, we expect this language is easy to learn.

Recent catastrophic events are stark reminders of the global implications of crisis management technology that can cope with nondeterministic environments. This is

${ }^{*}$ The research reported here is part of the Interactive Collaborative Information Systems (ICIS) project, supported by the Dutch Ministry of Economic Affairs, grant nr: BSIK03024. 
because major incidents generally entail much informational and operational chaos [7]. Emergency response of such events involved collaborations of people from different backgrounds, roles and professions. The need of a standard representation to reduce the ambiguity and multitude of semantic interpretation of human observers' reports becomes more apparent. Moreover, due to potentially overloaded or destroyed wired-communication infrastructures, these observers may only rely on their mobile devices (e.g. PDAs) with wireless connection for communication. However, the user interaction options for a PDA are quite limited along with decreasing size of this device. Moreover, for adding speech recognition capabilities to a PDA, a close to mouth microphone is necessary. The environment in which the technology is used must be similar to the training environment of the system [3], whereas PDAs are often used in various environments under various conditions. The result is misrecognition of commands, which is frustrating to the user. This leads us to aim at a natural interaction style based on GUI using an icon-based interface for communication.

In earlier work, we have investigated icons in particular for a languageindependent context application. We have developed an icon-based interface for communication in crisis situations on a PDA [11]. A user can report about crisis situations using spatial arrangements of icons. The use of icons to represent concepts or ideas makes user interactions on the interface particularly suitable across user diversity and differences between organizations and actors involved in the crisis management. However, a PDA represents a typical device, where a user has to carry out interaction tasks in a varying environment, with a small device and usually by using only one hand. The situation often requires multitasking, where the attention cannot be devoted fully on inputting. In demanding situations, e.g. crisis situations, usability and improvement in the input method performance are highly desired. Using our interface, users will likely interact with a large number of icons. To enhance the interface for usable and faster icon selections, we introduce an adaptive icon menu.

We have developed a method for adapting the icon menu layout adopted from the Fitaly keyboard [21]. The most relevant icons to a user's input context are placed on or around the center. Usability of the experimental implementation was examined with user tests. Verifiable measurement data and comments of test users were collected in order to guide further development.

This paper reports the basic idea of our adaptive icon-based interface. It is structured as follows. The following section starts with the related work. Then, the developed icon-based interface, which is deployed on a serious game environment, is presented. The next section presents our adaptive icon menu. We continue with describing our experiments. Finally, we report our conclusion.

\section{Related Work}

Semiotics approach provides theories and methods for designing and analyzing signs and symbols that can be understood universally. Recent attempts have been done in developing computer-based icon-based communication, for example: the Hotel Booking System [26], CD-Icon [2], Sanyog [1], and the Elephant's memory [15]. Most of these systems are too complex to learn or language-specific. A deep research has been done on designing such system that allows people to communicate with each 
other when they share no common language [22]. The system is based on the notion of simplified speech by reducing complexity and non-linear order. This is possible because as a visual language, sequencing and ordering may not be necessary.

In the field of crisis management, a comprehensive evaluation of existing symbols (or icons) for sharing information during crucial emergency situations has been performed by Dymon (2003) [7]. The US Government has promoted these symbols for emergency response applications on a national basis [13]. These symbols have been tested nationally with participations from private and public sectors. An iconbased interface for sharing and merging topological maps in damaged buildings has also been developed by Tatomir et al. (2006) [30]. The constructed map can be used for providing navigations or rescue action coordination.

Pen-based text entry on PDAs has been studied extensively [24]. Previous work in developing adapted keyboard layouts for PDAs and single-handed use has concentrated on alternative key configuration for improving entry speed, for example: Metropolis [33] and OPTI [25]. Fitaly keyboard (Fig. 1(a)) used an ad hoc optimization approach to minimize the distance between common character pairs [21]. The resulting keyboard contains two space bars and the letters are arranged so that common pairs of letters are often on neighboring keys. According to [24], this layout is one of the "fastest" for expert users.

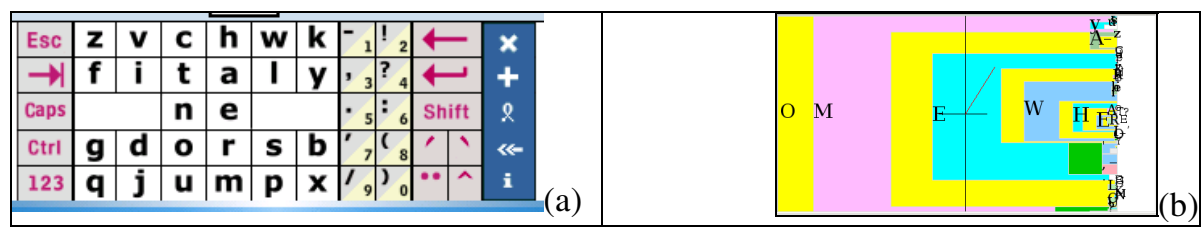

Fig. 1. (a) Fitaly keyboard [21] and (b) Dasher [33]

Some text input techniques have been developed with both movement minimizing and predictive features. T9 text entry works by comparing sequences of key presses to a stored database of possible words [31]. Dasher (Fig. 1(b)) employs continuous input by dynamically arranging characters in multiple columns positioning the next most likely character near the user's stylus [33]. The options are presented to the user in boxes sized according to their relative probabilities, to optimize the movement time. Our approach adopts these techniques for searching-minimizing and predicting input.

\section{System Overview}

\subsection{Icon-Based Interface}

We designed an icon-based interface for supporting people who must work collaboratively using knowledge from geospatial information and coordinate actions for resolving crisis. These people are professionals (rescue workers in the crisis field and crisis center room operators) and civilians (victims and witnesses). The interface provides icons, geometrical features, icon sentences, and text and photo selections for 
describing a crisis situation. A user can select and place them on a map where the crisis is occurring (see Fig. 2(a)). Sensed data, what the user sees, hears, smells, and even feels or experiences, can be transformed into icon representations. Geometrical features, e.g. arrow, ellipse, rectangle, can be used to indicate an area and highlight or emphasize an object, an event or a location on the map. Besides providing icons for representing observations, such as explosion, victim, ambulance, the interface also provides icons for reporting non-spatial information using text, photos, and 2D icon sentences. A pop-up window will appear when the user selects these types of icons.

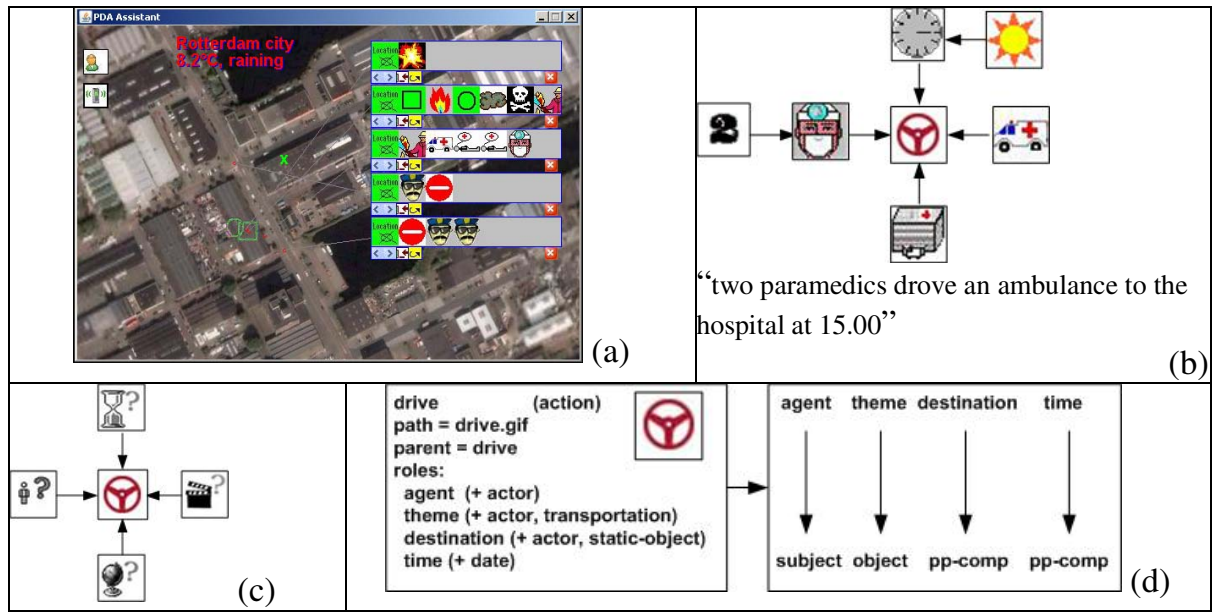

Fig. 2. The developed icon-based interface, (b) an example of two-dimensional icon sentences (15.00 is distinguished by the icon "noon" - represented by the image "sun"), (c) hints for the concept "drive", and (d) a schematic view of a case for the concept "drive"

Fig. 2(b) shows an example of 2D icon sentences. Inspired by Deikto [6], a user can construct an acyclic-graph of icons to represents a sentence. The graph connects icons using arrows, in which the former icon explains the latter. The sentence construction has no linear order. The user may start the string from anywhere she/he wants but if a verb is selected, the structure of the sentence will be determined.

We develop ontology by defining a case (i.e. a set of attributes) for each icon our ontology. For example: the icon "victim" contains number, location and status. For icons that represent verbs, we defined their case based on the theory of Fillmore [9] and VerbNet [19]. Each verb in ontology has a link with associated thematic roles (e.g. an agent, patient, and theme) and syntactic arguments (e.g. subject, object, and preposition) (Fig. 2(d)). Using this approach, the interface can give hints, which attributes can be filled in (Fig. 2(c)). As the icon is deselected, the hint will disappear. This hint can be selected and replaced by an icon to form a sentence. The approach gives a freedom to users to fill in the parts of a sentence, but at the same time the system can restrict the choices of icons which lead to a meaningful sentence.

The system uses Lexicalized Tree Adjoining Grammar (LTAG - [17]) to convert the icon sentence into text. Based on the case of every icon in a $2 \mathrm{D}$ string, a parser 
processes the $2 \mathrm{D}$ stream and maps each thematic role into the elementary trees of TAG families. The interface gives direct feedback on the user's selections. Therefore, although some icons are still unknown, the users can learn them on trials.

To help users find their way around a large number of icon vocabularies, we grouped related icons based on their semantic meaning. The icon vocabularies are displayed in a hierarchy tabular menu. The first version of the interface also provides an n-gram-based next icon prediction tool, which predicts and ranks most likely icons to follow a given segment of icon selections. The next version combined both approaches to be one icon menu layout (see section 4).

We took some guidelines and standards to guide us during our design process, such as: ISO/IEC [16], Horton's [14] and Schneiderman's [29]. Since the interpretation of icons is a subjective matter, in particular, we have tested each icon in the context of other icons based on Horton's [10]. To solve problems of linguistics and culturally bias, the test participants were selected to include different nationalities.

\subsection{Serious Game Environment}

We designed a simulation environment to facilitate the testing of the proposed interface. The simulation offers integration with real observations of a crisis situation. In this simulation, a human user plays the role of an observer using the developed icon-based interface on a PDA. Fig. 3(a) shows an overview of the simulator. In a real world, a crisis simulation plays out. In an observer world, the observers report their observations to a crisis center using their PDA. The data set of the real world and the observer world are geo-referenced overlay one another. The crisis center has an expert system to give most probable causes and advice about the current situation. For example: if the chances of fire are increased, the emergency is alerted and firemen are sent. The simulator receives the users' reports and updates the activities in the disaster simulator.

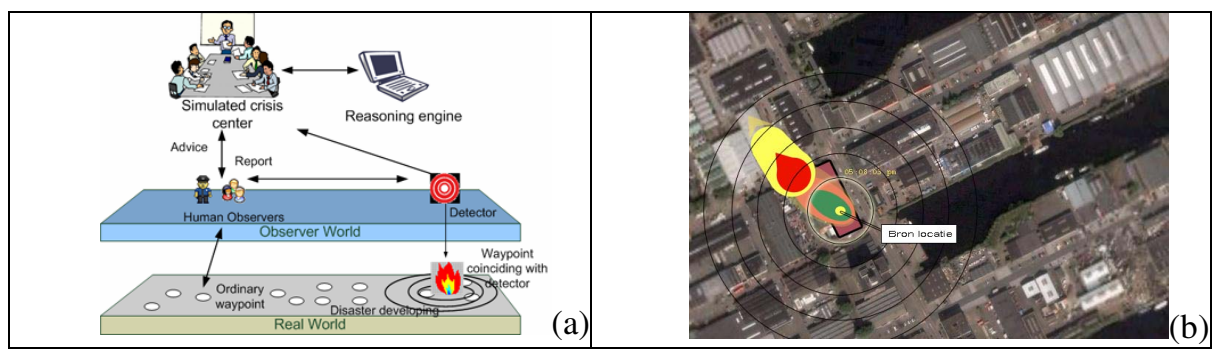

Fig. 3. (a) Overview of the simulation environment and (b) an example of a gas dispersion scenario shown by the simulator's interface

The geospatial knowledge of the real world is represented using grids and vectors that contain information about the environment. The simulator uses the vector values to model physical phenomena and its impact, such as spreading fire, gas dispersion, and spread of a hazardous material (Fig. 3(b)). The simulation is generated based on a scripted scenario. This scenario is a set of snapshots taken at every time unit on 
certain vector points. Each snapshot is advanced every minute. To enable real and mobile users to participate in a simulated reality, we provide knowledge of the virtual world. The current version of the implementation simulator is able to display an image of a situation relevant to the current scenario based on the location of an agent.

\section{Fitaly-Based Icon Menu}

Fig. 4(c) shows the Fitaly-based icon menu. The key ideas are the center placement of the most relevant icons to the current input context and different sizes of icons (the center menu has bigger icons than the outer). It has 28 icons and the double oversized icon "!" (to emphasis the message) and icon "?". In addition, two columns at the right side in the Fig. 4(c) are always and only available for constructing messages on a map. For constructing 2D icon sentences, they consist of numbers $0 . .9$. The adaptive layout is formed in three sequence steps.

Firstly, the method uses an ontology-driven approach to bridge the heterogeneity of crisis domain context. As described above, we have represented domain knowledge of each icon in the system's ontology, which is stored as w3c-OWL [32]. The ontology represents context that binds verbal thought (to remember or recall) and icons together. The ontology provides a natural way to group icons based on their concepts. It also provides information about relations of a concept to other concepts.

We distinguish two algorithms in forming the menu layout based on user's task. For constructing icon-based messages on a map, the algorithm is based on the taxonomy of the concepts that indicates relevancy between concepts contextually. For example: the icon "fire" is closer contextually to "firemen" from the same superconcept "fire-event" than to "collision" from "accident-event". For creating icon sentences, the algorithm depends on the state of the sentence construction. In a new sentence construction, the algorithm uses the taxonomy information to display groups of icons. After the first icon is selected, it uses information about the relation between concepts. This information can be derived from the properties of the selected concept in the ontology. For the following sentence(s), this algorithm prioritizes icons that are closer contextually to the previous sentence based on the taxonomy information.

These algorithms ensure that the suggestion icons are contextually and conceptually relevant with the user's input so far. To measure the distance between concepts, based on [18], these algorithms calculate the smallest number of synonymy (synset) steps between the semantic representation of two direct linked concepts in the ontology (= MPL value) by employing WordNet [8]. Using this information, the distance $\left(C_{\mathrm{i}}\right)$ of an icon to the context of the one(s) that is currently selected by the user, is the summation of the MPL values of interlinked concepts.

In the second step, we use statistical models of a language and a predictive engine that increases icon throughput by guessing or suggesting what the user will select next. The probability of an icon sentence is estimated using Bayes rule:

$$
P^{\prime}(s)=P\left(w_{1}, w_{2}, \ldots, w_{n}\right) .1 / C_{i}=\prod_{i=1}^{n} P\left(w_{i} \mid w_{1}, \ldots, w_{i-1}\right) .1 / C_{i}=\prod_{i=1}^{n} P\left(w_{i} \mid h_{i}\right) .1 / C_{i}
$$

where $h_{\mathrm{i}}$ is the relevant history when predicting $w_{\mathrm{i}}$ and $C_{\mathrm{i}}$ is the distance of the concept of $w_{\mathrm{i}}$ to the concept of $h_{\mathrm{i}}$. To predict the most likely icon in a given context, a 
global estimation of the icon sentence probability is computed by estimating conditional probabilities of $\mathrm{n}$-grams type features. This approach needs a large amount of data to compute multi-grams model. The current implementation collects the data from user selections during interaction. For training, it uses the database of the previous version of the interface. Additionally, the system records whether the icon is really the intended one or the user points it by mistakes. This information can be derived from the corrections that the user makes before a message is sent.

Finally, the method arranges the most probable 28 icons following the order shown in Fig. 4(b) (starts from no. 1). The system constantly re-arranges the icons dynamically based on the prediction.

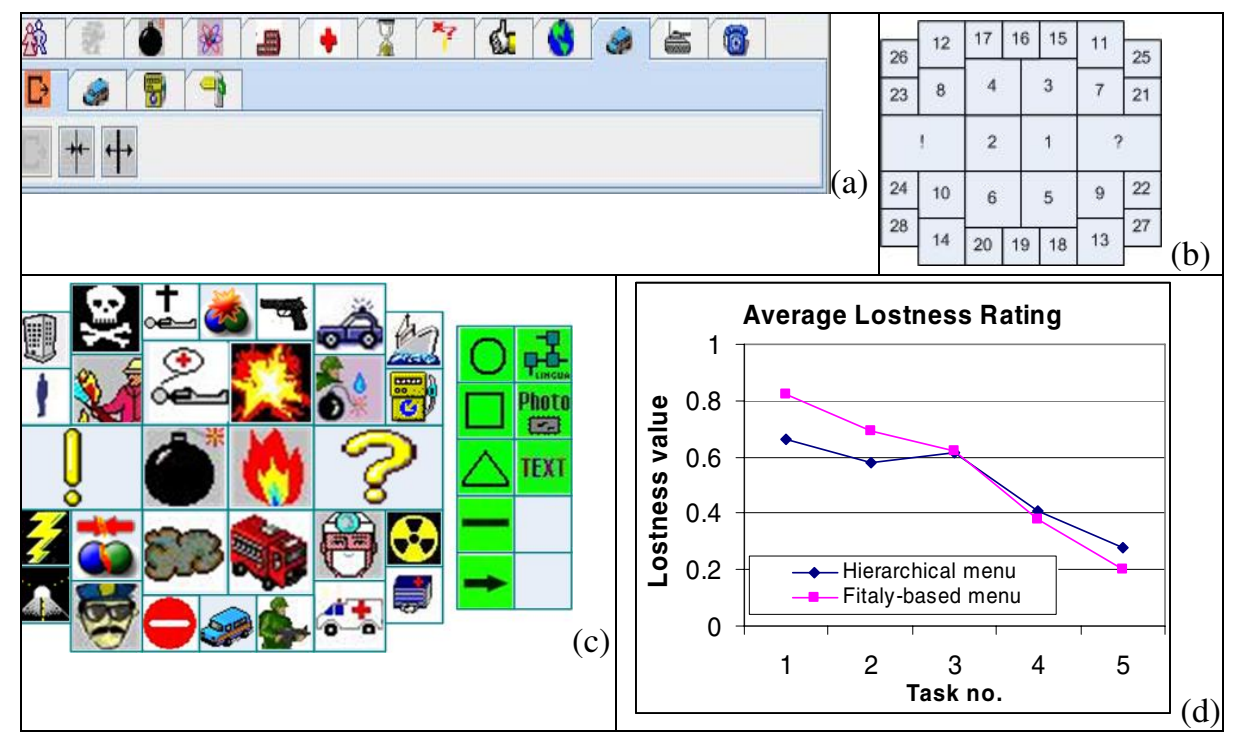

Fig. 4. (a) The hierarchical icon menu, (b) the ordering of icons, (c) the (adopted) Fitaly-based icon menu after a user selects "explosion", and (d) the average lostness rating

\section{Evaluation}

A set of experiments was performed to assess whether or not users were still able to create messages using provided icons from the Fitaly-based icon menu. It addressed the usability on interacting with the new interface. For this purpose, we compare the use of the hierarchical tabular icon menu (using the previous version - Fig. 4(a)) and the use of the Fitaly-based menu. Eight people took part in the test and one person as a pilot. They were selected in the range age of 25-50 years old. Four people used the hierarchical menu; the other used the Fitaly based menu. They were asked to walk around in certain locations. The tasks were created based on a crisis scenario that was fed into the simulator. The simulator used images of real crisis situations, which were sent to the participants based on their location in the world. The participants were asked to report what they saw or might sense using the icon-based interface on their 
PDA. After the experiments, we interviewed their satisfaction. All interactions on the interface were logged and the interviews were recorded on a tape recorded, to be analyzed afterward. Fig. 5 shows an example of data for analysis.

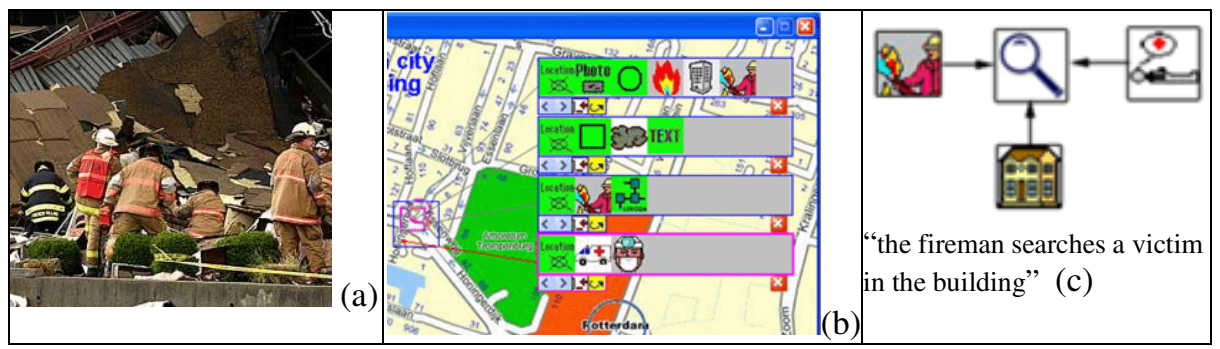

Fig. 5. Evaluation data: (a) an example of an image of a real crisis situation, (b) an example of icon-based message on a map and (c) an example of a $2 \mathrm{D}$ icon sentence created by a user

Smith's measurement of the sense of being lost in hypermedia [28] was adapted to measure disorientation (being lost) in an icon-based interface. As our icon space was made up by interlinked icons, we thought that an icon-based interface also might give cognitive overload and disorientation to its users. The problem referred to "after some trials, users cannot find what they are looking for". We compared the number of user selections whilst searching to the ideal path. For a perfect search, the lostness rating should have been equal to 0 . From the results, it appeared that all users were able to express their concepts and ideas using icons. All participants could report crisis situations for the given images. The experimental results also indicate that our target users (using both interfaces) still had problems in finding their desired icons. This was indicated by the high number of the lostness rating of the first five sequence tasks (see Fig. 3(d)). One of the reasons was referring to problems in recognizing some icons. Another problem is caused of the limited icon vocabularies.

In the first few sessions, the lostness rating of the Fitaly-based menu was higher than the hierarchical menu's. The reason was that the proposed vocabulary was not the one that the users intended. It appeared that the test users tend to keep searching for the icon they intended. If they could not find their desired icon, time was needed to find more relevant concept to represent their message. The participants should have rethought other concepts that could fit with the current problem domain they faced. This usually occurred when our participants tried to familiarize with the interface.

All participants accomplished their tasks with relevant icon-based messages for given images. The result of the remaining tasks shows that the lostness rating of the Fitaly menu was lower than the hierarchical menu's. We viewed this decreasing lostness rating in terms of improvement of user performances. From the interview, it appeared that after few tasks, these users thought that the menu became smarter. The icon selection became faster and easier. We concluded that our test users only needed a small period of time to adapt to both interfaces. 


\section{Discussion and Conclusion}

An experimental communication interface on a PDA has been developed, which is dedicated for reporting crisis situations. To reduce the ambiguity and the different semantic interpretations of human observers' reports, we have proposed collaborating information using icons. As icons offer a potential across language barriers, user interaction on the interface particularly is suitable in national and global aspects of crisis management. Users can describe a situation using icons, geometrical features, icon sentences, images and text on a map-based interface. It has been tested in a simulation environment that is able to capture and adapt the interactions between people and the interface in a scenario based on the users' reports.

We have developed an adaptive icon menu layout according to its usage and the user input context for faster and easier icon finding. Due to the novelty of the idea, we start with studying its qualitative characteristics to optimize its performance. From the experimental results, it appears that our target users are able to express concepts and ideas in their mind using a spatial arrangement of icons. However, the results also indicate that adaptation time is required, better icon designs and more icon vocabularies are necessary. In the first few tasks, users of the adaptive menu showed worse performance than the hierarchical menu. The reason is referring to the automatic result of the next selection prediction. The test users tended to keep searching instead of accepting the suggestions. In the following tasks, the users showed more familiar with the interface with better performance. The results indicated our test users had taken benefits provided by the menu layout and the prediction method.

The primary results show that the adaptive menu approach offers a usable tool to investigate. However, conforming MacKenzie et al. [24], it is potentially efficient and fast for expert users, but it may be difficult and slow for novice users due to the visual search requirement. Therefore, the interface should give the users both options of menu layouts to choose. Besides for more quantitative test results, future work should be done to gather data about how people might use the interface and how they experience in real crisis situations.

\section{References}

1. Basu, A., Sankar, S., Chakraborty, K., Bhattarcharya, S., Choudhury, M., Patel, R.: Vernacula Education and Communication Tool for the People with Multiple Disabilities. In: Development by Design Conference, Bangalore (2002)

2. Beardon, C.: CD-Icon, an Icon-based Language-Based on Conceptual Dependency. In: Intelligent Tutoring Media, 3(4), (1992)

3. Bousquet-Vernhettes, C., Privat, R., Vigouroux, N.: Error Handling in Spoken Dialogue Systems: Toward Corrective Dialogue. In: ISCA'03, USA (2003)

4. Chang, S.K., Polese, G., Orefice, S., Tucci, M.: A Methodology and Interactive Environment for Icon-based Language Design. Int. Journal of Human Computer Studies 41, 683-716 (1994)

5. Corballis, M.C.: Did language Evolve from Manual Gestures? In: 3rd Conf. of The Evolution of Language'00, France (2000)

6. Crawford, C.: Erasmatron. (2005), http://www.erasmatazz.com.s

7. Dymon, U.J.: An Analysis of Emergency Map Symbology. Int. Journal of Emergency Management 1(3), 227-237 (2003) 
8. Fellbaum, C.: WordNet: An Electronic Lexical Database. The MIT Press, Cambridge (1998)

9. Fillmore, C.J.: The Case for Case, in Universals in Linguistic Theory. In: Emmon Bach and Robert Harms, Holt, Rinehart and Winston: New York (1968)

10. Fitrianie, S.: An Icon-Based Communication Tool on a PDA. In: Postgraduate Thesis, TU Eindhoven, the Netherlands (2004)

11. Fitrianie, S., Datcu, D., Rothkrantz, L.J.M.: Constructing Knowledge of the World in Crisis Situations using Visual Language. In: IEEE SMC'06, pp. 121-126 (2006)

12. Frutiger, A.: Sign and Symbols, Their Design and Meaning. Van Nostrand Reinholt: New York (1989)

13. Homeland Security Working Group. In: Symbology Reference. (2003), http://www. fgdc.gov/HSWG

14. Horton, W.: The Icon Book. John Wiley, New York (1994)

15. Housz, T.I.: The Elephant's Memory (1994-1996), http://www.khm.de/ timot

16. ISO/IEC. International Standard, Information Technology - User System Interfaces and Symbols - Icon Symbols and Functions, 1st edn, ISO/IEC 11581-1:2000(E) to ISO/IEC 11581-6:2000(E) (2000)

17. Joshi, A.K., Levy, L., Takahashi, M.: Tree Adjunct Grammars. Journal of the Computer and System Sciences 10, 136-163 (1975)

18. Kamps, J., Marx, M.: Words with Attitude. In: Global WordNet CIIL'02, India (2002)

19. Kipper-Schuler, K.: VerbNet: A Broad-Coverage, Comprehensive Verb Lexicon. In: PhD thesis proposal, University of Pennsylvania (2003)

20. Kjeldskov, J., Kolbe, N.: Interaction Design for Handheld Computers. In: APCHI'02, Science Press, China (2002)

21. Langendorf, D.J.: Textware Solution's Fitaly Keyboard V1.0 Easing the Burden of Keyboard Input. In: WinCELair Review (1988)

22. Leemans, N.E.M.P.: VIL, A Visual Inter Lingua. In: Doctoral Dissertation, Worcester Polytechnic Institute, USA (2001)

23. Littlejohn, S.W.: Theories of Human Communication. 5th edn. Wadsworth (1996)

24. MacKenzie, I.S., Soukoreff, R.W.: Text Entry for Mobile Computing: Models and Methods, Theory and Practice. Human-Computer Interaction 17, 147-198 (2002)

25. MacKenzie, I.S., Zhang, S.X.: The Design and Evaluation of a High-Performance Soft Keyboard. In: ACM CHI, pp. 25-31. ACM, New York (1999)

26. Mealing, S., Yazdabi, M.: Communicating Through Pictures. In: Department of Computer Science, University of Exeter, England (1992)

27. Perlovsky, L.I.: Emotions, Learning and Control. In: International Symposium: Intelligent Control, Intelligent Systems and Semiotic, pp. 131-137 (1999)

28. Smith, P.: Toward a Practical Measure of Hypertext Usability. Interacting with Computers, Elsevier Science Ltd B.V. 8(4), 365-381 (1996)

29. Schneiderman, B.: Designing the User Interface: Strategies for Effective Human-Computer Interaction, 2nd edn. Addison-Wesley Publishing, London (1992)

30. Tatomir, B., Rothkrantz, L.J.M.: Intelligent System for Exploring Dynamic Crisis Environments. In: Van de Walle, B., Turoff, M. (eds.) ISCRAM'06. NJ, SA (2006)

31. Tegic Communication, T9. (1998), http://www.t9.com/faq.html

32. W3C, OWL: Ontology Web Language, http://www.w3.org/TR/owlguide

33. Ward, D.A., Blackwell, A., MacKay, D.: Dasher - a Data Entry Interface Using Continuous Gesture and Language Models. In: UIST'00, pp. 129-136 (2000)

34. Zhai, S., Hunter, M., Smith, B.A.: The Metropolis Keyboard - An Exploration of Quantitative Techniques for Virtual Keyboard Design. In: ACM UIST, pp. 119-128. ACM, NY (2000) 